\title{
Cardiomiopatía periparto. Levosimendán en la insuficiencia cardiaca aguda
}

\author{
Peripartum cardiomyopathy. Levosimendan in acute heart failure \\ Rafael Espinosa, ${ }^{*}$ Erik Vidal, ${ }^{\ddagger}$ Belén Jacinto, ${ }^{\ddagger}$ Juan Pablo Vázquez, ${ }^{\ddagger}$ \\ Jesús Vázquez, § Samuel Karchmer
}

Citar como: Espinosa R, Vidal E, Jacinto B, Vázquez JP, Vázquez J, Karchmer S. Cardiomiopatía periparto. Levosimendán en la insuficiencia cardiaca aguda. Acta Med Grupo Angeles. 2021; 19 (3): 372-375. https://dx.doi.org/10.35366/101730

\section{Resumen}

Este caso muestra la respuesta terapéutica de levosimendán en una mujer con insuficiencia cardiaca aguda secundaria a cardiomiopatía periparto. Mediante ecocardiografía se demuestra la rápida recuperación de la función ventricular izquierda, y el descenso del péptido natriurético cerebral. Este medicamento inotrópico es el primer agente disponible de los conocidos como sensibilizadores del calcio, con propiedades vasodilatadoras y que influyen directamente en la recuperación de la función ventricular izquierda en la insuficiencia cardiaca aguda.

Palabras clave: Postparto, cardiomiopatía, levosimendán.

\section{INTRODUCCIÓN}

La cardiomiopatía periparto (CMPP) es la insuficiencia cardiaca aguda, grave, que afecta a las mujeres en los últimos meses del embarazo o en la etapa inicial del puerperio. En 1971, Demakis y su equipo definieron los criterios para el diagnóstico de insuficiencia cardiaca en el último mes del embarazo, o seis meses después de parto, en ausencia de alguna causa identificable de insuficiencia cardiaca, $y$ antes del último mes del embarazo. Los criterios ecocardiográficos para el diagnóstico son: fracción de expulsión menor a $45 \%$, fracción de acortamiento ventricular izquierdo menor a 30\%, y diámetro diastólico final del ventrículo izquierdo

\footnotetext{
* Cardiólogo.

‡ Terapia Intensiva.

$\S$ Neumólogo.

" Ginecoobstetra.
}

Hospital Ángeles Lomas. Estado de México.

\section{Abstract}

This case shows the therapeutic response of levosimendan in a woman with acute heart failure secondary to postpartum cardiomyopathy. Through echocardiography she demonstrated rapid recovery of the left ventricular function and decreased brain natriuretic peptide. This is the first inotropic medication agent available from so-called calcium sensitizers with vasodilatory properties that directly influence the recovery of left ventricular function in acute heart failure.

Keywords: Postpartum, cardiomyopathy, levosimendan.

mayor de $2.7 \mathrm{~cm} / \mathrm{m}^{2}$ de superficie corporal. ${ }^{1,2}$ Se excluyen causas congénitas y adquiridas de insuficiencia cardiaca y la diferencian de otras causas de insuficiencia cardiaca. ${ }^{3}$ En el tratamiento de la insuficiencia cardiaca aguda, las drogas inotrópicas son fundamentales; en este caso, el levosimendán fue el medicamento que contribuyó a la normalización rápida de la función ventricular izquierda.

\section{REPORTE DE CASO CLÍNICO}

Mujer de 39 años de edad con antecedentes de un aborto y una cesárea. Cursó con hipertensión arterial sistémica relacionada al embarazo. En la semana 39 se le

Correspondencia:

Dr. Rafael Espinosa

Correo electrónico: respinosacardiogia@hotmail.com

Aceptado: 02-09-2020.

www.medigraphic.com/actamedica

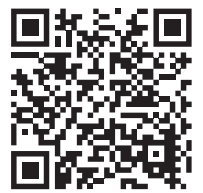


practicó cesárea; después de 12 horas inicia con disnea súbita y desaturación por pulsioximetría. Se auscultan en ambos pulmones abundantes estertores intersticiales. En la radiografía de tórax se observa importante hipertensión vascular pulmonar (Figura 1). Con oxígeno suplementario se ingresa a la Unidad de Terapia Intensiva. La tensión arterial fue 150/89 $\mathrm{mmHg}$, frecuencia cardiaca 130 latidos por minuto, frecuencia respiratoria de 26 por minuto, temperatura $36.5{ }^{\circ} \mathrm{C}$, saturación de oxígeno por pulsioximetría $\left(\mathrm{SpO}_{2}\right) 81 \%$ con aporte de oxígeno. El primer ecocardiograma transtorácico (ETT) muestra la fracción de expulsión del ventrículo izquierdo (FEVI) de 40\%, presión sistólica de la arteria pulmonar de $43 \mathrm{mmHg}$, sin alteraciones regionales de la contracción. El péptido natriurético cerebral (BNP) fue 1,527 pg/mL, el dímero $\mathrm{D}$ de 4,100 U. El tratamiento inicial fue con ventilación mecánica no invasiva (VMNI), furosemida, captopril y dobutamina en dosis $8 \mu \mathrm{g} / \mathrm{kg} / \mathrm{h}$. A las 48 horas las manifestaciones de insuficiencia cardiaca se agravan, el ETT mostró FEVI de 33\% (Figura 2). Al tratamiento se

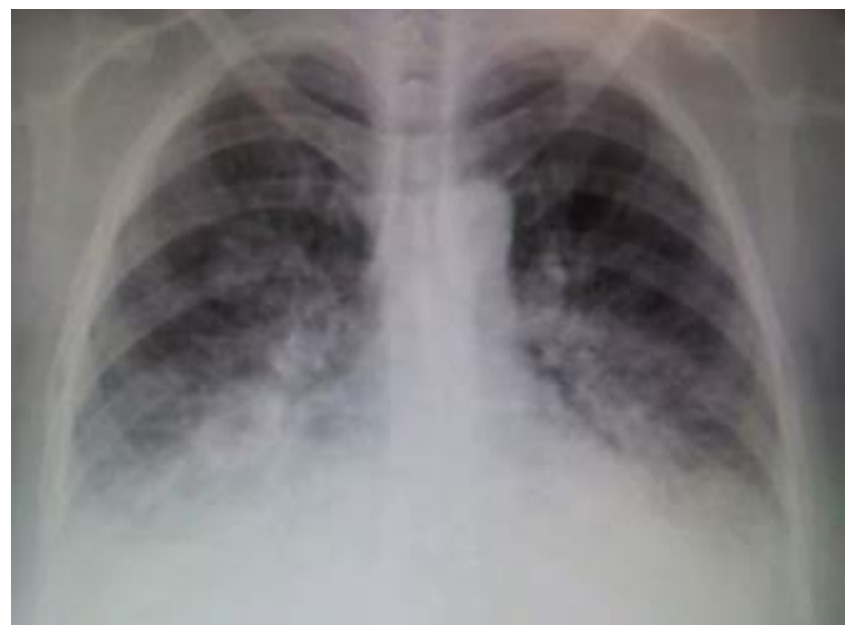

Figura 1: Radiografía de tórax. Se observa hipertensión venocapilar pulmonar. Sin cardiomegalia. adiciona levosimendán en dosis de bolo de $10 \mu \mathrm{g} / \mathrm{kg} / 10$ minutos, seguido de infusión continua de $0.2 \mu \mathrm{g} / \mathrm{kg} / \mathrm{min}$ por 24 horas. Evoluciona con mejoría, la saturación venosa central pasó de 61 a 73\%, una hora después de iniciado el tratamiento, el ETT mostró FEVI de $44 \%$ y a las 24 horas de 56\%, este resultado correlaciona con la disminución progresiva del BNP en forma importante.

En la evolución a los 10 meses se encuentra en clase funcional I, y el ETT muestra FEVI de 68\% (Figura 3).

\section{DISCUSIÓN}

La incidencia de la CMPP se presenta de 1:300 a 1:4,000 embarazos, es provocada por causas genéticas y/o culturales. ${ }^{4}$ Otros factores relacionados con la enfermedad pueden ser: embarazos múltiples, historia familiar, tabaquismo, diabetes mellitus, hipertensión arterial sistémica, malnutrición, madres de edad avanzada o uso prolongado de beta agonistas. ${ }^{5}$

La etiología parece tener implicaciones infecciosas, inflamatorias, autoinmunes, y también se ha asociado con la hormona prolactina.

La manifestación más importante es la insuficiencia cardiaca por disfunción sistólica del ventrículo izquierdo durante el final del embarazo o en las primeras semanas después del parto. No deben existir otras causas de disfunción del ventrículo izquierdo, y aunque el ventrículo no esté dilatado, la fracción de expulsión deberá estar por debajo de $45 \%$.

La mortalidad se reporta de 20 a $85 \%$ en las pacientes. ${ }^{6,7}$

Aunque la presentación más frecuente es la insuficiencia cardiaca aguda, también pueden manifestarse arritmias ventriculares complejas.

Aun con manejo apropiado de la insuficiencia cardiaca aguda, ésta puede evolucionar rápidamente hacia el deterioro y el fallecimiento.

En nuestro caso, a pesar del tratamiento inotrópico con dobutamina en forma aguda, hubo deterioro grave de la función ventricular manifestada con edema pulmonar; se
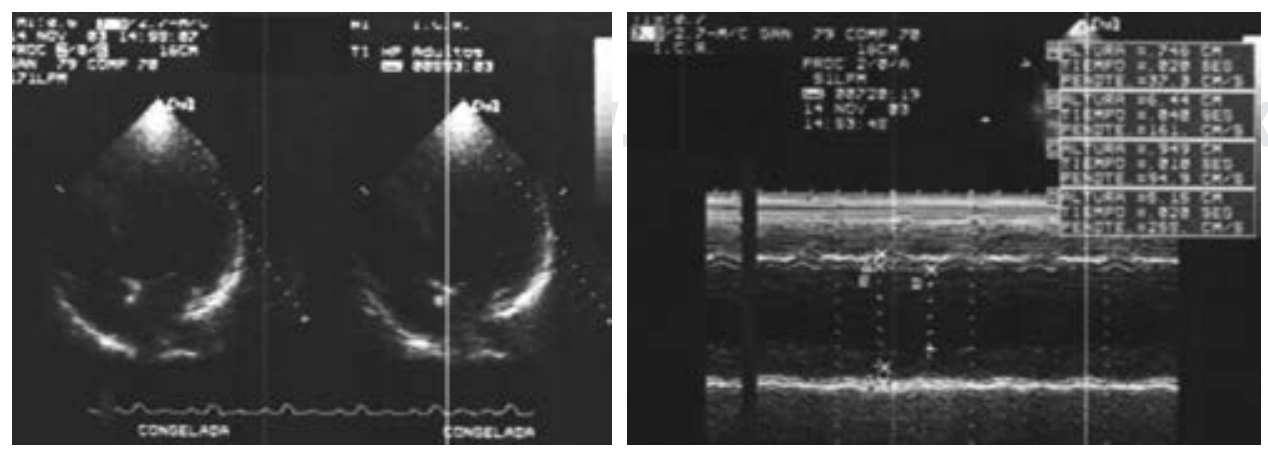

Figura 2:

Ecocardiograma transtorácico con fracción de expulsión del ventrículo izquierdo de $33 \%$. 


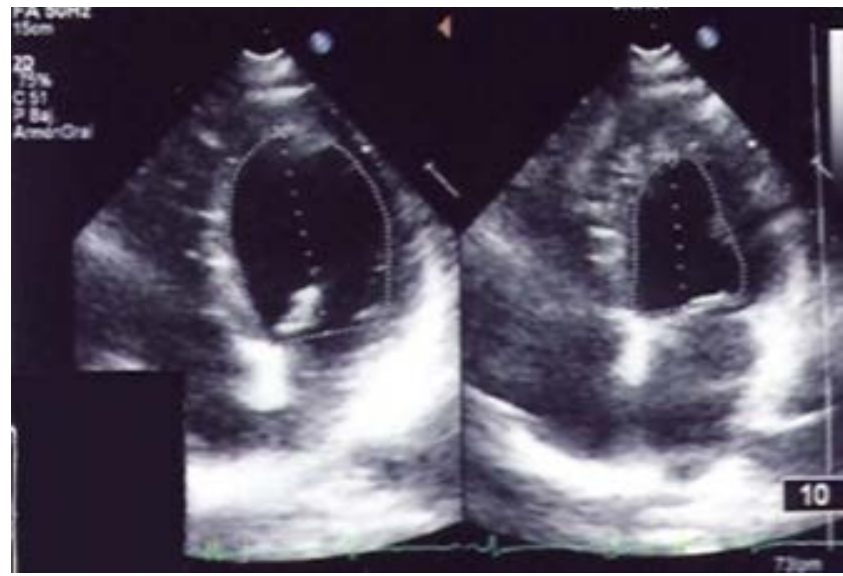

Figura 3: Ecocardiograma transtorácico cuatro meses después con fracción de expulsión del ventrículo izquierdo de $68 \%$.

adicionó levosimendán y a los 20 minutos se presentó la mejoría clínica, corroborada por el BNP y por el ETT.

El levosimendán es el primer agente disponible de la clase de medicamentos conocidos como sensibilizadores del calcio, éstos mejoran la contractilidad miocárdica sin incremento significativo del calcio intracelular, aumentando la sensibilidad de los miofilamentos sensibles al calcio durante la sístole, lo cual aumenta la fuerza y la contracción ventricular, y mejora el gasto cardiaco sin incrementar el consumo de oxígeno. Esta droga puede mejorar la relajación diastólica y provoca vasodilatación en los lechos coronarios, pulmonares y sistémicos. ${ }^{8}$ Como se ha reportado, las pacientes responden satisfactoriamente a esta droga. ${ }^{9-12}$

Este medicamento impulsa la rápida mejoría hemodinámica que se acompaña de alivio de los síntomas, sin estar asociado a importantes efectos adversos. ${ }^{10}$ La dosis recomendada es iniciar con un bolo en 10 minutos de 6 a $24 \mu \mathrm{g} / \mathrm{kg}$, y continuar con infusión de 0.05 a $0.2 \mu \mathrm{g} / \mathrm{kg} /$ min, estas dosis son bien toleradas $i^{13}$ por arriba de dichas dosis la frecuencia de efectos colaterales aumenta, como hipertensión arterial, náuseas y cefalea.

Es superior al placebo y a la dobutamina en pacientes con insuficiencia cardiaca crónica. Algunos estudios han sugerido que puede mejorar la eficiencia mecánica del ventrículo derecho. Tampoco se ha confirmado su uso en choque cardiogénico. ${ }^{10}$

Los efectos de levosimendán comparados con dobutamina en pacientes con insuficiencia cardiaca severa, muestran que fue mejor en la recuperación de la función hemodinámica. Este beneficio se acompañó de menor mortalidad a los 180 días. $^{10}$

En contraste, con otro estudio comparativo entre levosimendán y dobutamina en pacientes con insuficiencia cardiaca aguda, se registró mayor descenso de BNP en el grupo de levosimendán, pero sin disminución significativa en la mortalidad a 180 días. $^{14}$

De acuerdo con nuestro resultado y los reportes relacionados con la CMPP, se puede recomendar este medicamento en el inicio del tratamiento.

Sólo hay una referencia en la literatura de esta patología tratada con este inotrópico, en donde se muestra el resultado sin diferencias a las que estamos reportando. ${ }^{11}$

Aunque son pocos los reportes, podemos aportar que este medicamento en la CMPP con insuficiencia cardiaca aguda, contribuye a la rápida recuperación de la función ventricular y a la recuperación de la clase funcional; el inicio temprano del tratamiento integral, en especial con los medicamentos inotrópicos, fue lo que nos permitió que se prolongara y progresara la disfunción ventricular.

\section{CONCLUSIÓN}

La CMPP es una enfermedad poco frecuente y muy grave. El diagnóstico de la insuficiencia cardiaca es clínico y ecocardiográfico, con evidencia de disfunción ventricular. El tratamiento es intensivo con la combinación de digoxina, diuréticos, betabloqueadores, vasodilatadores, inhibidores de la enzima convertidora de la angiotensina, inotrópicos y anticoagulantes. En algunas ocasiones se puede tratar con inmunodepresores e inmunoglobulinas. En los casos más graves es necesario el soporte circulatorio mecánico y trasplante cardiaco. El pronóstico está relacionado con la prontitud en el inicio del tratamiento médico y con la recuperación de la función ventricular izquierda.

\section{REFERENCIAS}

1. Demakis JG, Rahimtoola SH. Peripartum cardiomyopathy. Circulation. 1971; 44 (5): 964-968.

2. Demakis JG, Rahimtoola SH, Sutton GC, Meadows WR, Szanto PB, Tobin JR et al. Natural course of peripartum cardiomyopathy. Circulation. 1971; 44 (6): 1053-1061.

3. Hibbard JU, Lindheimer M, Lang RM. A modified definition for peripartum cardiomyopathy and prognosis based on echocardiography. Obstet Gynecol. 1999; 94 (2): 311-316.

4. Fett JD, Christie LG, Carraway RD, Murphy JG. Five-year prospective study of the incidence and prognosis of peripartum cardiomyopathy at a single institution. Mayo Clin Proc. 2005; 80 (12): 1602-1606.

5. Elkayam U, Akhter MW, Singh H, Khan S, Bitar F, Hameed A et al. Pregnancy-associated cardiomyopathy: clinical characteristics and a comparison between early and late presentation. Circulation. 2005; 111 (16): 2050-2055.

6. Heider AL, Kuller JA, Strauss RA, Wells SR. Peripartum cardiomyopathy: a review of the literature. Obstet Gynecol Surv. 1999; 54 (8): 526-531.

7. Pearson GD, Veille JC, Rahimtoola S, Hsia J, Oakley CM, Hosenpud JD et al. Peripartum cardiomyopathy: national heart, lung, and blood institute and office of rare diseases (National Institutes of Health) 
workshop recommendations and review. JAMA. 2000; 283 (9): 1183-1188.

8. Lehtonen LA. Levosimendan: a parenteral calcium-sensitising drug with additional vasodilatory properties. Expert Opin Investig Drugs. 2001; 10 (5): 955-970.

9. Follath F, Cleland JG, Just H, Papp JG, Scholz H, Peuhkurinen K et al. Efficacy and safety of intravenous levosimendan compared with dobutamine in severe low-output heart failure (the LIDO study): a randomised double-blind trial. Lancet. 2002; 360 (9328): 196-202.

10. Slawsky MT, Colucci WS, Gottlieb SS, Greenberg BH, Haeusslein E, Hare J et al. Acute hemodynamic and clinical effects of levosimendan in patients with severe heart failure. Study Investigators. Circulation. 2000; 102 (18): 2222-2227.
11. Benlolo S, Lefoll C, Katchatouryan V, Payen D, Mebazaa A. Successful use of levosimendan in a patient with peripartum cardiomyopathy. Anesth Analg. 2004; 98 (3): 822-824.

12. Nguyen HD, McKeown B. Levosimendan for post-partum cardiomyopathy. Crit Care Resusc. 2005; 7 (2): 107-110.

13. Nieminen MS, Akkila J, Hasenfuss G, Kleber FX, Lehtonen LA, Mitrovic $\checkmark$ et al. Hemodynamic and neurohumoral effects of continuous infusion of levosimendan in patients with congestive heart failure. J Am Coll Cardiol. 2000; 36 (6): 1903-1912.

14. Mebazaa A, Nieminen MS, Packer M, Cohen-Solal A, Kleber FX, Pocock SJ et al. Levosimendan vs dobutamine for patients with acute decompensated heart failure: the SURVIVE Randomized Trial. JAMA. 2007; 297 (17): 1883-1891. 